\title{
Near-field enhanced imaging by a magnetized ferrite slab
}

\author{
R. Marqués, ${ }^{\text {a) }}$ F. Mesa, and F. Medina \\ Departamento de Electrónica y Electromagnetismo, Facultad de Física, Universidad de Sevilla, \\ Av. Reina Mercedes s/n, 41012 Sevilla, Spain
}

(Received 3 May 2004; accepted 17 November 2004; published online 4 January 2005)

\begin{abstract}
This letter demonstrates the near-field imaging enhancement at microwave frequencies of two-dimensional sources by a ferrite slab magnetized to saturation. It is shown that this effect is based on the nonreciprocal amplification of magnetostatic surface waves (MSSW) across the ferrite slab. The inclusion of losses in our analysis has also made it possible to prove this effect for realistic yttrium iron garnet ferrite samples. For ferrite slabs of width $d$, the resolution at the image plane (at a distance $2 d$ from the source) is better than the resolution in air at a distance $d$ of the source, which leads to an equivalent air length of the ferrite slab less than zero. Since the constitutive parameters of saturated ferrites depend on the external magnetizing field, the operation frequency of the proposed imaging devices can be tuned by varying this biasing field. (c) 2005 American Institute of Physics. [DOI: 10.1063/1.1852091]
\end{abstract}

The prediction of the perfect lens effect in a left-handed slab ${ }^{1}$ has certainly grown an increasing interest on the focusing properties of materials with negative parameters. In addition to focusing at intermediate-field distances, ${ }^{2}$ it has been shown that improved near-field imaging can be achieved by means of media with only negative permittivity, such as metals at optical frequencies. ${ }^{1,3}$ This behavior has been found to be a consequence of the coupling of surface plasmons at both slab interfaces. ${ }^{1}$ Spin waves with a behavior similar to surface plasmons in metals can be found in ferrimagnetic slabs at microwave frequencies. This fact suggests the possibility of obtaining some kind of near-field imaging enhancement by employing these materials. Extremely low-loss ferrite slabs are presently available as microwave materials. Since losses strongly degrade the image enhancement, ${ }^{4}$ this last property of ferrites can result in a significant improvement of the imaging. Another advantage would be the tunability of the frequency of operation, which could be achieved by varying the intensity of the external magnetizing field.

Cubic low-loss soft ferrites, such as ytrium iron garnets (YIGs), are commonly manufactured for diverse applications in microwave technology. When they are magnetized to saturation by an external static magnetic field $\mathbf{H}_{0}=H_{0} \hat{\mathbf{z}}$. its magnetic properties are well described by the so-called Polder tensor $^{5}$

$$
\overline{\bar{\mu}}=\left(\begin{array}{ccc}
\mu & -i \kappa & 0 \\
i \kappa & \mu & 0 \\
0 & 0 & 1
\end{array}\right),
$$

with

$$
\mu=1+\frac{\omega_{m} \omega_{h}}{\omega_{h}^{2}-\omega^{2}} ; \kappa=\frac{\omega \omega_{m}}{\omega_{h}^{2}-\omega^{2}},
$$

where $\omega_{h}=\gamma H_{0}$ and $\omega_{m}=4 \pi \gamma M_{s}$ ( $\gamma$ is the gyromagnetic ratio, and $M_{s}$ is the magnetization of saturation). Magnetic losses can be accounted for by the substitution $\omega_{h} \rightarrow \omega_{h}$ $+(i / 2)\left(\Delta H / H_{0}\right) \omega$, where $\Delta H$ is the resonance linewidth. Magnetostatic or nonexchange spin waves are well known

${ }^{\text {a)} E l e c t r o n i c ~ m a i l: ~ m a r q u e s @ u s . e s ~}$ solutions of the electromagnetic wave equation in magnetic media characterized by Eq. (1). They are called magnetostatic because most of their energy is associated with the magnetic field. Therefore, the electric field can be neglected in a first order of approximation and $\nabla \times \mathbf{H} \approx \mathbf{0}$. Thus, $\mathbf{H} \simeq-\nabla \phi_{m}$, where $\phi_{m}$ is the magnetostatic potential, which must satisfy the magnetostatic wave equation, $\nabla \cdot \bar{\mu} \cdot \nabla \phi_{m}$ $=0$, or

$$
\left[\mu\left(\frac{\partial^{2}}{\partial x^{2}}+\frac{\partial^{2}}{\partial y^{2}}\right)+\frac{\partial^{2}}{\partial z^{2}}\right] \phi_{m}=0 .
$$

Magnetostatic surface waves (MSSWs) are those specific solutions of the magnetostatic wave Eq. (3) that are confined to the surface of the ferrite. ${ }^{5}$ For ideal lossless ferrites, they appear when $\mu+\kappa=-1$, or equivalently for a frequency $\omega$ $=\omega_{h}+\frac{1}{2} \omega_{m}$. In this case, the magnetostatic potential takes the form $\phi_{m}=\phi_{0} \exp (-i \omega t+i k y \pm k x)$, decaying exponentially at both sides of the ferrite interface [see Fig. 1(a)]. Therefore, MSSWs are similar to surface plasmons in many aspects. However, due to the anisotropy of the magnetized ferrite, they only propagate at the specific direction defined by the wave vector $\mathbf{k}=k \hat{\mathbf{k}}$, with $\hat{\mathbf{k}}=\hat{\mathbf{H}}_{0} \times \hat{\mathbf{n}}$ [see Fig. 1(a)], but not in the opposite one. This behavior turns out to be a direct consequence of the violation of the Lorentz reciprocity theorem in magnetized ferrites. ${ }^{5}$

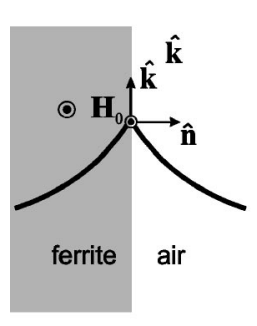

(a)

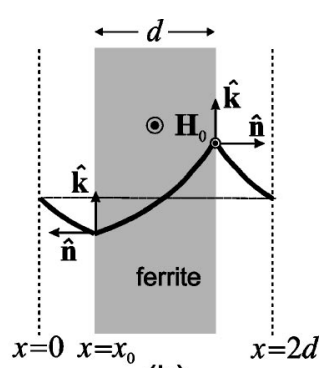

(b)
FIG. 1. (a) Representation of the amplitude of a MSSW at a ferrite interface (solid curve). The directions of the magnetizing field $\mathbf{H}_{0}$, the propagation unit vector $\hat{\mathbf{k}}$, and the surface normal $\hat{\mathbf{n}}$ are illustrated. (b) Representation of the amplitude of a MSSW in a quasiperfect near-field ferrite focusing device. 
Let us now consider the extension of the solution of Eq. (3) sketched in Fig. 1(a) to a ferrite slab of finite width $d$ as in Fig. 1(b). The solution sketched in Fig. 1(a) is a physical solution, decaying at both sides of the ferrite interface. However, there is also a mathematical solution of Eq. (3) that exponentially grows at both sides of the ferrite interface and propagates along the direction $\hat{\mathbf{k}}=\hat{\mathbf{n}} \times \hat{\mathbf{H}}_{0}$ (see Ref. 5). This latter solution can be imposed at the left interface of the slab and combined with the former one at the right interface to give the solution sketched in Fig. 1(b). This field distribution shows exactly the behavior needed for the onset of the perfect lens effect, ${ }^{1}$ and becomes physical if the appropriate source is located at $x=0$. In fact, the amplitude of the MSSWs at $x=0$ [see Fig. 1(b)] is restored at $x=2 d$ for all the wave numbers $k$. Next, it will be considered a twodimensional time-harmonic magnetic field source located at $x=0$, at a distance $x_{0}<d$ from the left slab interface. If $d$ and $x_{0}$ are both much smaller than the free-space wavelength associated with the source frequency, we are in the realm of the magnetostatic approach. Thus, the magnetic field produced by the earlier source can be expressed as the following Fourier integral of magnetostatic waves (propagating at both directions along the $y$ axis and decaying towards the ferrite slab):

$$
\phi_{m}(x, y) \approx \frac{1}{2 \pi} \int_{-\infty}^{\infty} \widetilde{\phi}_{m}(k) e^{i k y-k x-i \omega t} \mathrm{~d} k,
$$

where $\widetilde{\phi}_{m}(k)$ is the Fourier transform of $\phi_{m}(y)$. Following the earlier theory, after setting up a source frequency equal to the frequency of the MSSWs $\left(\omega=\omega_{h}+\frac{1}{2} \omega_{m}\right)$, the amplitude of all the magnetostatic waves propagating along the positive $y$ direction will be restored at a distance $2 d$ from the source [see Fig. 1(b)]. On the contrary, the magnetostatic waves propagating along the negative $y$ direction will be reflected by the slab, and hardly reproduced at the right-hand side of the slab. Assuming a near zero amplitude for these latter magnetostatic waves at $x=2 d$ [see Fig. 1(b)], the magnetostatic field amplitude at $x=2 d$ will be approximately given by

$$
\phi_{m}(2 d, y) \approx \int_{0}^{\infty} \widetilde{\phi}_{m}(k) e^{i k y-i \omega t} \mathrm{~d} k
$$

or

$$
\phi_{m}(2 d, y) \approx \frac{1}{2} \phi_{m}(0, y)+\frac{i}{2 \pi} \int_{-\infty}^{\infty} \frac{\phi_{m}(0, u)}{y-u} \mathrm{~d} u,
$$

where $\phi_{m}(0, y)$ is the magnetostatic potential at the source plane, and the second integral term can be identified as the Hilbert transform of this latter quantity. ${ }^{6}$ If, for instance, the magnetic field distribution at the source plane is given by a delta function, the corresponding magnetic field at $x=2 d$ - the image plane-will be a delta function plus the Hilbert transform of this function (the $1 / y$ function). This example illustrates how the ferrite slab behaves as a quasiperfect near-field focusing device.

Up to now only ideal lossless ferrites have been considered in the frame of the magnetostatic approach. However, actual ferrites are lossy, which substantially affects the focusing properties of the ferrite slab. ${ }^{4}$ Moreover, the magnetostatic approximation is strictly valid only in the limit $d \rightarrow 0$. In order to have a complete picture of the aforementioned

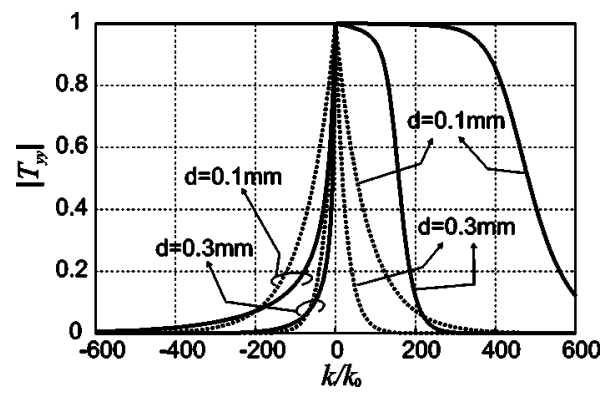

FIG. 2. Magnitude of the $y y$ element of the transfer matrix as a function of the normalized wave vector $k / k_{0}\left(k_{0}=\omega / c\right)$ for a ferrite slab as that shown in Fig. 1(b), with $x_{0}=d / 2$ and constitutive parameters $\epsilon_{r}=12,4 \pi M_{s}=1200 \mathrm{G}$, and $\Delta H=1 \mathrm{Oe}$. The external magnetizing field is $H_{0}=520 \mathrm{Oe}$, and the operation frequency is $f=3.1351 \mathrm{GHz}$. Curves for $d=0.1$ and $0.3 \mathrm{~mm}$ are shown (solid lines). For comparison purposes, the values of the transfer functions in air at a distance $2 d$ of the source are also shown (dashed lines)

effects in actual ferrite slabs, an exact electromagnetic analysis has been also performed. This analysis accounts for both propagating and evanescent waves in the Fourier expansion of the fields [at a difference of (4-6)]. It also accounts for magnetic losses by introducing a nonvanishing resonance linewidth $\Delta H$ in the Polder tensor (1). Assuming a given distribution of the transverse-to- $x$ magnetic field, $\mathbf{H}_{t}(0, y) \exp (-i \omega t)$, at the source plane $(x=0)$, the magnetic field at the image plane $(x=2 d)$ can be expressed as the following spectral-domain integral:

$$
\mathbf{H}_{t}(2 d, y)=\frac{1}{2 \pi} \int_{-\infty}^{\infty}[\mathbf{T}(k ; \omega)] \cdot \widetilde{\mathbf{H}}_{t}(0, k) e^{i k y-i \omega t} \mathrm{~d} k,
$$

where $\tilde{\mathbf{H}}_{t}(0, k)$ is the Fourier transform of $\mathbf{H}_{t}(0, y)$, and $[\mathbf{T}(k ; \omega)]$ is a spectral-domain transfer matrix. This transfer matrix is obtained after solving the Maxwell equations with the appropriate boundary conditions at the source plane, at the air-ferrite interfaces, and for $x \rightarrow \infty$ (Sommerfeld radiation condition). Thus, assuming an $\exp [i(k y-\omega t)]$ phase factor common to all the fields, the transverse-to- $x$ fields can be related by $\mathbf{X}(x, k)=[\mathbf{A}(k)] \cdot \mathbf{X}(0, k),{ }^{7,8}$ where array $\mathbf{X}$ $=\left(E_{y}, E_{z}, H_{y}, H_{z}\right)$ and $[\mathbf{A}]$ is a $(4 \times 4)$ matrix that only depends on the characteristics and dimensions of the ferrite and air layers. The combination of the earlier relation with the free space boundary condition at the image plane, leads to the following expression for the transfer matrix:

$$
\begin{aligned}
{[\mathbf{T}]=} & \left\{\left[\mathbf{Z}_{0}\right]-\left[\mathbf{A}_{11}\right]\left[\mathbf{A}_{21}\right]^{-1}\right\}^{-1} \cdot\left\{\left[\mathbf{A}_{12}\right]-\left[\mathbf{A}_{11}\right]\right. \\
& \left.\times\left[\mathbf{A}_{21}\right]^{-1}\left[\mathbf{A}_{22}\right]\right\},
\end{aligned}
$$

with $\left[\mathbf{A}_{i j}\right]$ being the $(2 \times 2)$ submatrices of matrix $[\mathbf{A}]$ and $\left[\mathbf{Z}_{0}\right]$ the free-space impedance matrix. Following the aforementioned procedure, the $T_{y y}$ term of the transfer dyad for a typical low-loss YIG slab has been computed, and the results are shown in Fig. 2.

The transfer matrix in air, at a distance $2 d$ from the source plane, is also shown in Fig. 2. The transfer matrix for the ferrite slab is nearly unity for positive values of $k$. This behavior is maintained up to a maximum value of $k$, which increases for thinner slabs. This result is consistent with the reported theory (the thinner the slab, the better the magnetostatic approximation works). It clearly suggests a significant improvement of the resolution in the presence of the ferrite slab. In order to illustrate this latter point, the image of an imposed magnetic field at the source, $H_{y}(0, y)$, is shown in 


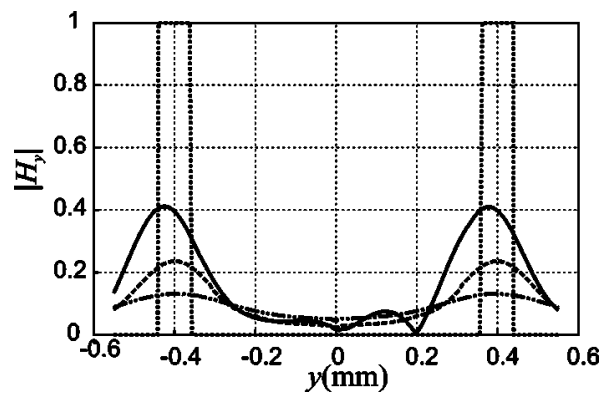

FIG. 3. The image of an imposed magnetic field at the source plane $(x$ $=0$ ) of Fig. 1(b) given by $H_{y}=1$ in the strips and zero otherwise (dotted line). The value of the $H_{y}$ field at the image plane $(x=2 d)$ is shown (solid line). The images in air at a distance $x=d$ (dotted line), and at a distance $x=2 d$ (dot-dashed line) are also shown. Constitutive parameters of the ferrite are as in Fig. 2, with $d=0.1 \mathrm{~mm}$.

Fig. 3. The imposed magnetic field is unity at two thin strips (marked in the figure) and zero otherwise. A significant enhancement of the image through the ferrite can be clearly seen. As usual, the image through the ferrite has been compared with the value of the $H_{y}$ field in air, at a distance $2 d$ from the source. This comparison shows that the equivalent air length of the ferrite is less than $d$. In addition, the image through the ferrite has been also compared with the image through air, at a distance $d$ from the source. This latter comparison is of great interest, since it shows that the equivalent air length of the ferrite slab is less than zero.

Near-field imaging using MSSWs in ferrite slabs has several advantages over other methods. One of them is the reduced level of losses, which provides a significant improvement of the imaging. ${ }^{4}$ In fact, it is possible to find commercial YIG slabs providing ratios $\Delta H / H_{0}$ of a few hun- dredths. Another interesting advantage of the present proposal is tunability. Resolution enhancement in slabs made of materials with negative parameters is restricted to a single frequency (that providing the adequate values for the constitutive parameters). Therefore, devices made with this kind of materials will be usually restricted to a single frequency of operation. However, since the constitutive parameters of ferrites strongly depends on the external magnetizing field, the frequency of operation of imaging ferrite devices can be tuned by varying the magnitude of this externally applied field. The price to pay for these advantages is that, owing to the anisotropy inherent to magnetized ferrites, the aforementioned over-resolution can be only achieved in the plane perpendicular to the magnetizing field [that shown in Fig. 1(b)]. Another inherent limitation of focusing devices based on the reported effect is that they could only operate in the nearfield limit, with ferrite slabs of width $d \leqq 0.01 \lambda_{0}$, where $\lambda_{0}$ is the free space wavelength.

This work has been supported by the Spanish Ministry of Science and Technology and FEDER funds (Project Nos. TEC2004-03214 and TEC2004-04249-C02-02).

${ }^{1}$ J. B. Pendry, Phys. Rev. Lett. 85, 3966 (2000).

${ }^{2}$ A. Grbic, and G. V. Eleftheriades, Phys. Rev. Lett. 92, 117403 (2004).

${ }^{3}$ J. T. Shen, and P. M. Platzman, Appl. Phys. Lett. 80, 3286 (2002).

${ }^{4}$ R. Marqués and J. Baena, Microwave Opt. Technol. Lett. 41, 290 (2004).

${ }^{5}$ A. G. Gurevich, and G. A. Melkov Magnetization Oscillations and Waves (CRC, Boca Raton, FL, 1996).

${ }^{6}$ S. S. Haykin, Communication Systems (Wiley, New York, 1983).

${ }^{7}$ C. M. Krowne, IEEE Trans. Microwave Theory Tech. 32, 1617 (1984).

${ }^{8}$ F. Mesa and M. Horno, IEEE Trans. Microwave Theory Tech. 43, 233 (1995). 